\title{
Aspetti medico-legali dei trapianti d'organo in Italia
}

\author{
Paolo Bruzzone ${ }^{1}$ \\ Dipartimento di Chirurgia Generale, Specialità Chirurgiche e Trapianto d'Organo "Paride Stefanini” della Prima Facoltà di Medicina e Chirurgia. \\ Università La Sapienza, Roma

\begin{abstract}
In the last decade new immunosuppression strategies have been developed, with the introduction of new pharmacological agents that act on different stages of the cell cycle (including for example sirolimus and brequinar). Nevertheless, in transplantation management there are still many controversies concerning medicolegal aspects. This article examines the Italian normative on transplantation, underlining the debate about the meaning of the term "brain death" as a description of the clinical condition of patients' status as living or dead.
\end{abstract}

Keywords: transplantation, brain death, total brain failure

Medicolegal aspects of organ transplantation in Italy

Pratica Medica \& Aspetti Legali 2009; 3(2): 75-78

\section{INTRODUZIONE}

I trapianti di organi solidi hanno presentato un marcato miglioramento quantitativo e qualitativo nel corso degli anni '80, come conseguenza:

- del perfezionamento delle tecniche chirurgiche impiegate sia nel donatore e nel ricevente sia nella chirurgia di banco;

- del miglioramento delle metodiche di preservazione ipotermica grazie alla messa a punto della soluzione Università di Wisconsin, che ha permesso di prolungare sensibilmente i tempi di ischemia fredda per rene, fegato e pancreas;

- dell'evoluzione dei protocolli di terapia immunosoppressiva per merito di ciclosporina, introdotta in Italia dal nostro Centro nel 1982, e di altri farmaci quali sirolimus (RS 61443), everolimus e brequinar (FK 506) (Tabella I).

Quest'ultima molecola ha dimostrato una cospicua efficacia nel trattamento del rigetto cronico dopo trapianto di fegato e nella prevenzione del rigetto acuto e cronico nel trapianto di intestino, anche in associazione con altri organi [1].

\begin{tabular}{|ll|}
\hline \multicolumn{1}{|c|}{ Principio attivo } & \multicolumn{1}{c|}{ Indicazioni } \\
\hline Sirolimus & $\begin{array}{l}\text { Prevenzione del rigetto nel trapianto di rene } \\
\text { allogenico }\end{array}$ \\
\hline Ciclosporina & $\begin{array}{l}\text { Prevenzione del rigetto di trapianto } \\
\text { allogenico di rene, fegato, cuore, cuore- } \\
\text { polmone, polmone e pancreas }\end{array}$ \\
\hline Brequinar* & $\begin{array}{l}\text { Prevenzione del rigetto acuto e cronico } \\
\text { nel trapianto di intestino, trattamento del } \\
\text { rigetto cronico dopo trapianto di fegato }\end{array}$ \\
\hline Azatioprina & $\begin{array}{l}\text { Profilassi del rigetto nel trapianto di } \\
\text { organi allogenici quali rene, fegato, cuore, } \\
\text { polmone e pancreas }\end{array}$ \\
\hline Tacrolimus & $\begin{array}{l}\text { Profilassi del rigetto nel trapianto di organi } \\
\text { allogenici quali rene, fegato e cuore }\end{array}$ \\
\hline Everolimus & $\begin{array}{l}\text { Profilassi del rigetto nei pazienti sottoposti } \\
\text { a trapianto renale o cardiaco allogenico }\end{array}$ \\
\hline (acido micofenolico) & $\begin{array}{l}\text { Profilassi del rigetto acuto in pazienti che } \\
\text { ricevono un allotrapianto renale, cardiaco } \\
\text { o epatico }\end{array}$ \\
\hline & \\
\hline
\end{tabular}

Tabella I. Principali farmaci immunosoppressori e loro indicazioni * non in commercio in Italia 


\section{ASPETTI LEGISLATIVI E ORGANIZZATIVI}

È noto che il prelievo degli organi solidi viene generalmente effettuato in donatori cadaveri il cui cuore è ancora battente, in condizioni quindi di "morte cerebrale", considerata definitiva e irreversibile. Laccertamento di questa condizione è stato sancito per la prima volta in Italia dalla legge $\mathbf{n}$. 644 del 2 dicembre 1975 e dal successivo regolamento n. 409 del 16 giugno 1977, che richiedevano un periodo di osservazione di 12 ore da parte di una Commissione medica.

Il dibattito scaturito nel corso degli anni ha comportato la discussione e la successiva approvazione della legge n. 578 del 29 dicembre 1993, il cui art. 1 ribadisce: «la morte si identifica con la cessazione irreversibile di tutte le funzioni dell'encefalo». In base all'art. 2 pertanto «la morte per arresto cardiaco si intende avvenuta quando la respirazione e la circolazione sono cessate per un intervallo di tempo tale da comportare la perdita di tutte le funzioni dell'encefalo».

Nei soggetti affetti da lesioni encefaliche sottoposti a misure rianimatorie la morte ha luogo "quando si verifica la cessazione irreversibile di tutte le funzioni dell'encefalo», accertata «da un collegio medico nominato dalla direzione sanitaria, composto da un medico legale o, in mancanza, da un medico di direzione sanitaria o da un anatomo-patologo, da un medico specialista in anestesia e rianimazione e da un medico neurofisiopatologo o, in mancanza, da un neurologo o da un neurochirurgo esperti in elettroencefalografia. I componenti del collegio medico sono dipendenti di strutture sanitarie pubbliche». Il legislatore introduce notevoli innovazioni, quali (art. 3) l'obbligo da parte del «medico della struttura sanitaria» di segnalare immediatamente tutti i casi di presunta morte nei soggetti affetti da lesioni encefaliche e sottoposti a misure rianimatorie «alla direzione sanitaria, che è tenuta a convocare prontamente il collegio medico".

Il decreto del Ministero della Sanità n. 582 del 22 agosto 1994 richiede (art. 3), per l'accertamento di morte nei soggetti affetti da lesioni encefaliche e sottoposti a misure rianimatorie, «la contemporanea presenza delle seguenti condizioni:

- stato di incoscienza;

- assenza di riflesso corneale, riflesso fotomotore, riflesso oculocefalico e oculovestibolare, reazioni a stimoli dolorifici portati nel territorio d'innervazione del trigemino, riflesso carenale e respirazione spontanea dopo sospensione della ventilazione artificiale fino al raggiungimento di ipercapnia accertata da $60 \mathrm{mmHg}$ con $\mathrm{pH}$ ematico minore di 7,40 ;
- silenzio elettrico cerebrale [...];

- assenza di flusso cerebrale preventivamente documentata».

Queste condizioni devono essere simultaneamente presenti "per almeno tre volte, all'inizio, alla metà e alla fine del periodo di osservazione» che, in base all'art. 4, non deve essere inferiore a:

- sei ore per gli adulti e i bambini di età superiore a cinque anni;

- dodici ore per i bambini di età compresa tra uno e cinque anni;

- ventiquattro ore nei bambini di età inferiore a 1 anno.

Il decreto del Ministero della Sanità del 18 marzo 1994 ha attribuito al Centro nazionale di riferimento per i trapianti, di cui all'art. 14 della legge n. 644, la funzione di «Coordinamento operativo nazionale delle attività di prelievo e trapianto di organi e tessuti».

Lattività legislativa è continuata negli anni successivi. Il Ministero della Sanità ha emanato il Decreto 8 aprile 2000 in tema di "Disposizioni in materia di prelievi e di trapianti di organi e di tessuti, attuativo delle prescrizioni relative alla dichiarazione di volontà dei cittadini sulla donazione di organi a scopo di trapianto" [2], cui è seguito, ad opera del Ministero della Salute, il Decreto 11 marzo 2008 in tema di "Integrazione del decreto 8 aprile 2000 sulla ricezione delle dichiarazioni di volontà dei cittadini circa la donazione di organi a scopo di trapianto" [3].

Il Ministero della Salute ha emanato il Decreto 2 dicembre 2004 sulle "Modalità per il rilascio delle autorizzazioni all'esportazione o all'importazione di organi e tessuti" [4].

Il Ministero della Salute ha infine emanato il Decreto 11 aprile 2008 "Aggiornamento del decreto 22 agosto 1994, n. 582 relativo al Regolamento recante le modalità per l'accertamento e la certificazione di morte" [5].

Il nuovo regolamento all'art. 4, comma 1, uniforma per i donatori di qualsiasi età, anche pediatrica e neonatale, la durata del periodo di osservazione, «violando in tal modo l'art. 4, comma 1, della legge n. 578 del 1993 in cui si afferma letteralmente la necessità di tener conto della peculiarità dei soggetti di età inferiore ai cinque anni. Già per questa ragione (ma ve ne sono anche altre) riteniamo che sia esperibile contro suddetto decreto un ricorso al TAR Lazio" [6].

A partire dagli anni ' 90 , in particolar modo negli Stati Uniti e in Spagna, si è riscontrato un ritorno a una pratica clinica che risale agli albori della trapiantologia, prima che il ben noto Comitato di Harvard introducesse il concetto di brain death, tradotto in italiano come "morte cerebrale" [7]. Si tratta del prelievo di organi dopo l'arresto cardiaco del donatore, cui eventualmente siano stati somministrati opportuni farmaci e nei cui vasi fe- 
morali siano stati posizionati pre-mortem speciali cateteri per la perfusione con soluzioni ipotermiche, da infondere dopo 1,5-5 minuti dall'arresto cardiaco. In alcuni casi si procede all'instaurazione di una vera e propria circolazione e ossigenazione extracorporea.

Non è il caso di dilungarsi oltre in questa sede su tali procedure, difficilmente realizzabili in Italia, dove per legge l'accertamento della morte per arresto cardiaco prevede l'osservazione del cadavere per 20 minuti con contemporanea esecuzione del tanatocardiogramma.

Comunque tali pratiche di prelievo multiorgano subito dopo l'arresto cardiaco hanno forse contribuito in parte a un "ripensamento" [8] del concetto di morte cerebrale, contribuendo in seguito a riaprire un dibattito che recentemente ha visto coinvolti anche alcuni autorevoli organi ed esponenti del Vaticano e della Chiesa Cattolica [9].

Già in passato alcuni Autori [10] avevano persino invocato, su base "utilitaristica", il superamento dell'attuale cardine etico della pratica trapiantologica, cioè della dead donor rule, che prevede l'illiceità del prelievo multiorgano in soggetti ancora vivi, dato che in questo caso l'intervento del chirurgo prelevatore causerebbe la morte del donatore.

Negli Stati Uniti è stato pubblicato nel dicembre 2008, da parte del President's Council on Bioethics, un white paper, cioè un dossier dal titolo "Controversies in the determination of death: a white paper by the President's Council on Bioethics" [11]: la maggioranza (ma non la totalità) dei suoi componenti ha rinnovato la fiducia ai criteri neurologici per l'accertamento della brain death, che tuttavia è stata rinominata, con una differenza semantica non irrilevante, total brain failure.

\section{CONCLUSIONI}

Non essendo mai stato applicato l'art. 4 legge 91/1999, i familiari del donatore in morte cerebra-

\begin{tabular}{|l|}
\hline - (Whole/total) brain death \\
\hline - Total brain failure \\
\hline - Coma dépassé \\
\hline - (Total) brain infarction \\
\hline - Irreversible apneic coma \\
\hline - Brain arrest \\
\hline
\end{tabular}

Tabella II. Terminologia adottata per definire la morte cerebrale nelle varie pubblicazioni. Total brain failure è il termine individuato come più preciso dal Council of Bioetics [11]

le in pratica possono ancora opporsi al prelievo di organi, a meno che non si proceda per legge ad autopsia o a riscontro diagnostico (art. 6 legge $n$. 644 del 2 dicembre 1975 e art. 10 del D.P.R. n. 409 del luglio 1977).

Altre Nazioni europee, come per esempio la Francia, hanno invece messo in pratica il principio del "consenso presunto" o del "silenzio-assenso": in base a esso si effettuano le operazioni di prelievo, una volta accertata la condizione di morte cerebrale, in tutti i pazienti deceduti in ospedale i quali non abbiano mai espresso in vita volontà contraria alla donazione. L'adozione di questi criteri incontra notevoli difficoltà di ordine psicologico e legislativo in numerosi Paesi, quali gli Stati Uniti, mentre in Italia potrebbe, secondo alcuni e in base a quanto avvenuto in altri Stati, non garantire automaticamente un incremento significativo degli organi utilizzabili a scopo di trapianto.

Il problema è dunque estremamente complesso e richiede ulteriori e articolate soluzioni sia dal punto di vista organizzativo e di informazione dell'opinione pubblica sia sul piano legislativo, con leggi di facile interpretazione e applicazione.

A tale proposito, alcune proposte di legge sul cosiddetto "testamento biologico" o "dichiarazione anticipata di volontà" prevedono espressamente che il firmatario del documento esprima chiaramente la propria volontà se donare o meno i suoi organi dopo il decesso.

\section{CODICE DI DEONTOLOGIA MEDICA (16 DICEMBRE 2006). CAPO IV: TRAPIANTI DI ORGANI, TESSUTI E CELLULE}

- Art. 38 - "Prelievo di parti di cadavere": «ll prelievo di parti di cadavere a scopo di trapianto terapeutico può essere effettuato solo nelle condizioni e nei modi previsti dalle leggi in vigore. II sostegno vitale dovrà essere mantenuto sino a quando non sia accertata la perdita irreversibile di tutte le funzioni dell'encefalo»

- Art. 40 - "Donazione di organi, tessuti e cellule": «È compito del medico la promozione della cultura della donazione di organi, tessuti e cellule anche collaborando alla idonea informazione ai cittadini» 


\section{BIBLIOGRAFIA}

1. Makowka L, Sher LS, Cramer DV. The development of brequinar as an immunosuppressive drug for transplantation. Immunol Rev 1993; 136: 51

2. Ministero della Salute. Decreto 8 aprile 2000. Gazzetta Ufficiale della Repubblica Italiana n. 89 del 15 aprile 2000; pp. 4-7

3. Ministero della Salute. Decreto 11 marzo 2008. Gazzetta Ufficiale della Repubblica Italiana n. 80 del 4 aprile 2008; pp. 15-6

4. Ministero della Salute. Decreto 2 dicembre 2004. Gazzetta Ufficiale della Repubblica Italiana n. 27 del 3 febbraio 2005; pp. 14-5

5. Ministero della Salute. Decreto 11 aprile 2008. Gazzetta Ufficiale della Repubblica Italiana n.136 del 12 giugno 2008; pp. 8-11

6. Barcaro R, Becchi P, Donadoni P. Prospettive bioetiche di fine vita. In: Becchi P. La morte cerebrale e il trapianto di organi. Milano: Franco Angeli, 2008; p. 12 (postilla)

7. Beecher, Henry K. A definition of irreversible coma. Report of the ad hoc Committee of the Harvard Medical School to examine the definition of brain death. JAMA 1968; 205: 337-40

8. Defanti CA. Soglie: medicina e fine della vita. Torino: Bollati Boringhieri, 2007

9. De Mattei R (a cura di). Finis Vitae. La morte cerebrale è ancora vita? Soveria Mannelli (CZ): Rubbettino, 2007

10. Truog RD. Is it time to abandon brain death? Hastings Center Report 1997; 27: 29-37

11. Council on Bioethics. Controversies in the determination of death: a white paper by the President's Council on Bioethics. Washington DC: Council on Bioethics, January 2009

\section{CORRESPONDING AUTHOR}

Dott.Paolo Bruzzone, e-mail:paolo.bruzzone@uniroma1.it 